\title{
Extracorporeal Shock Wave Therapy versus Low-Level Laser Therapy in the Management of Chronic Plantar Fasciitis
}

\author{
Gehad G. ELsehrawy', Samah I. Nasef ${ }^{*}$, Mostafa S. Ibrahim², Aziza S. Omar ${ }^{1}$ \\ Departments of ${ }^{1}$ Physical Medicine, Rheumatology and Rehabilitation, and ${ }^{2}$ Diagnostic Radiology, Faculty of \\ Medicine, Suez Canal University, Ismailia, Egypt
}

\begin{abstract}
Objectives: to compare and evaluate the effectiveness of extracorporeal shock wave therapy (ESWT) and low-level laser therapy (LLLT) using diagnostic ultrasound (US) in the management of chronic plantar fasciitis (PF). Patients and Methods: The study was done as a prospective, randomized, comparative clinical study. Patients with PF were randomly allocated into 2 groups: ESWT ( $n=23)$, LLLT $(n=23)$, group 1 underwent 2 sessions of ESWT (2050 shocks, 2.5 bars) and group 2 underwent 6 sessions of LLLT $(27 \mathrm{~J} / \mathrm{cm} 2 ; 830 \mathrm{~nm})$. All patients were assessed using the visual analog scale (VAS), foot function index (FFI) and diagnostic US to measure plantar fascia thickness and to assess echogenicity before and 1 month after treatment. Results: There were significant improvement after one month of treatment in the mean VAS, FFI, thickness of the plantar fascia and echogenicity in both groups. LLLT proved significantly superior to ESWT in pain relief ( $p=0.029$ ), but no significant difference between both groups in FFI improvement $(p=0.264)$. No statistically significant difference between both groups regarding improvement in US findings including plantar fascia thickness $(p=0.885)$ and echogenicity $(p=$ 0.34 ). Conclusion: LLLT proved to be more effective than ESWT in pain relief in patients with PF after one month of treatment.
\end{abstract}

Key Words: Extracorporeal shock wave, low-level laser therapy, plantar fasciitis, ultrasound

\section{Introduction}

Plantar heel pain is considered one of the most common pathologies of the foot, accounting for about $15 \%$ of foot- related complains presenting to physicians. The clinical manifestations can be disabling, and despite its high incidence, the specific cause of PF is poorly understood. It is likely that PF is multi-factorial and may be associated with local changes to the plantar fascia tissue, systemic disease, or altered ankle and foot biomechanics ${ }^{(1,2)}$. PF is thought to be due to biomechanical overuse from prolonged standing or running, consequently creating micro tears at the calcaneal enthesis. Some experts have considered this condition "plantar fasciosis" implying that its caused by a more chronic degenerative process versus acute inflammation $(3,4)$. The diagnosis of PF can be made with reasonable certainty 
on the basis of clinical assessment alone $^{(5)}$. However, US have recently received more attention because of its effectiveness in clinical settings. Sonography has been shown to provide valuable information about soft tissue including muscle, fascia, tendons, blood vessels, and nerves. It is believed to be convenient, low risk and low cost, and helping in the diagnosis and following problems associated with PF and to rule out other foot pathologies(6). Conservative therapies of PF including ice, taping, heel cups, night splints, stretching exercises, rest, heating modalities, therapeutic US, below-the- knee non weight bearing casts, and short-leg walking casts, besides local corticosteroid injection are effective in nearly $90 \%$ of patients $(7,8)$. Failure of these measures occurs in approximately $10 \%$ of patients, resulting in chronic PF which is a difficult clinical problem. There is also inadequate high-level of evidence guiding the management of this group of patients $^{(1)}$. Therapeutic laser offers a safe, effective, and easily utilized primary or adjunctive therapy that is relatively cost effective for clinicians \& patients. LLLT is more effective in pain relief and in the improvement of quality of life and functional ability thus LLLT can be an important adjunct especially in patients who develop intolerable adverse effects to drugs or invasive treatments ${ }^{(9)}$. LLLT has been shown to enhance proliferation of fibroblasts, and lymphocytes. Proliferation is thought to be due to photo-stimulation of the mitochondria leading to activation of signaling pathways and up regulation of transcription factors ultimately leading to increases in growth factors ${ }^{(10,11)}$. ESWT has been introduced for the treatment of chronic inflammatory and degenerative processes of bone-tendon junctions due to its ability to induce hyperemia, neovascularization, and regeneration of tendon tissues $^{(12)}$. Other hypothesized mecha- nisms of action of ESWT include the physical alteration of small axons, thus inhibiting pain impulse conduction; chemical alteration of pain receptor neurotransmitters, thereby reducing pain perception; and hyperstimulation activation of the gate control mechanism, thereby inducing analgesia(13). However, randomized controlled trials have reported conflicting results on whether ESWT alleviates the pain of recalcitrant PF patients ${ }^{(14,15)}$. Despite the increasing popularity of ESWT and LLLT in the treatment of chronic PF, there is no strong evidence to support the use of any of them and studies comparing the efficacy of these treatment modalities are limited ${ }^{(16)}$. So, the objectives of our study were to determine and compare the clinical effects and efficacy of ESWT and LLLT objectively using diagnostic US at 1 month of follow-up for patients with chronic PF.

\section{Patients and Methods}

From September 2016 to January 2018, the present study included 46 patients with chronic PF. All patients agreed to participate in the study after being informed about the aim of the study, examination steps, and treatment modalities application and each patient signed an informed consent. The medical ethics committee of the faculty of Medicine at Suez Canal University, Egypt approved the present study. This study was carried out as a prospective, randomized, comparative, clinical study in the Rheumatology and Rehabilitation department at Suez Canal University Hospital in Ismailia city, Egypt. Inclusion Criteria: Adults of both genders over 18 years were included. Diagnosis of painful heel syndrome were done by clinical examination, with the following positive clinical signs: Pain in the morning or after sitting a long time, Local pain where the fascia attaches to the heel and increasing pain with extended walking or 
standing for more than 15 minutes and history of 1 month of unsuccessful conservative medical treatment not including ESWT or LLLT. A minimum washout phase after preceding non-surgical treatments were required prior to enrollment in the study (a time gap of at least six weeks since the last corticosteroid injection; four weeks since the last local anesthetic injection, iontophoresis, therapeutic US, and one week since the last nonsteroidal antiinflammatory drugs). Patients included in the study were allowed to only take acetaminophen and they were asked to halt any other treatment modalities.

Exclusion Criteria: Patients were excluded from the study if they had neurological heel pain resulting from nerve entrapments, arthritis of the feet, traumatic heel pain, sensory neuropathies, peripheral vascular disease, local malignancies in the feet, autoimmune diseases, bleeding disorders, skin ulceration at the area of modality application, or cellulitis. Pregnant ladies and patients who were unable to fulfill follow-up criteria were also excluded.

Methods: All patients were assessed using the Visual Analogue Scale (VAS) score for heel pain before and after 1 month of treatment. Patients were asked to mark the severity of heel pain on one centimeter graded $10 \mathrm{~cm}$ line " 0 " indicating no pain and " 10 " indicating the worst pain imaginable. A decrease of three degrees or more in the VAS score was considered positive for patient improvement. The number of participants who attained a decrease of three degrees or greater in VAS score across the evaluation period was calculated for both subjects in the ESWT group and in the LLLT group as a proportion of the total number of subjects in each procedure group. Foot function was assessed in all patients using the foot function index (FFI) before and after 1 month of treatment. The FFI is a self- administered index consisting of 23 items divided into 3 sub-scales including Pain subscale, Disability subscale, and Activity Limitation subscale ${ }^{(17)}$. A decrease of $50 \%$ or more in the total FFI was considered positive for patient improvement. Following enrolment, screening, and baseline assessment, patients were randomized to either ESWT or LLLT. Each group included 23 patients. Group 1: Patients underwent ESWT (BTL-5000 Series) over the plantar aspect of the heel. The probe was oriented perpendicularly to the patient's heel, and US gel was used as a coupling agent. Ensuring the heel is in constant contact with the gel, the physician moves the heel in a slow, circular motion within the focal zone. Each patient received two sessions of ESWT with one-week interval that comprised 2050 shocks at a frequency of $10.0 \mathrm{~Hz}$ and intensity of 2.5 bars. Group 2: Patients underwent LLLT (BTL-4000 Series) $830 \mathrm{~nm}$ of laser light, at a frequency of $10.0 \mathrm{~Hz}$ leading to a total dose of 27.0 $\mathrm{J} / \mathrm{cm} 2$ was administered per session as: 2 procedures per week during a consecutive 3-week period with total 6 sessions. The laser probe was scanned into the areas of the painful heel, insertion of the plantar fascia on the medial calcaneal area. ESWT and LLLT therapy were performed by the same investigator. Both groups were educated to perform daily plantar fascia stretching, calf muscle stretching and Achilles tendon stretching exercises under physician supervision and at home while receiving ESWT or LLLT. Diagnostic ultrasound was performed for all patients before and 1 month after treatment to measure the thickness at the proximal plantar fascia where it attaches to the calcaneus and assess echogenicity.

Ultrasound protocol: Plantar fascia thickness and echogenicity was assessed using high resolution equipment (SONOACERS5, Samsung Medison), with a multi-frequency (5$12 \mathrm{MHz}$ ) linear transducer. Patients were 
examined in prone position with the foot hanging over the examination table, knee extended and ankle in $90^{\circ}$ dorsiflexion. The probe was placed over the plantar aspect of the hind foot in a longitudinal plane with adequate amount of gel applied in-between. Images obtained just medial to the midline at proximal end of plantar fascia distal to its origin from the medial tubercle of calcaneus. Thickness of the plantar fascia is measured at the thickest portion from the base of the medial calcaneal tubercle where a bright echogenic line was easily visible ${ }^{(18)}$. A perpendicular measurement was then taken to the top of the plantar fascia image where the most inferior border of the plantar fascia was discernable from fat. PF is diagnosed when the thickness of plantar fascia is greater than $4 \mathrm{~mm}$ or echogenicity is reduced or both ${ }^{(19)}$. A reduction in plantar thickness or change of echogenicity from hypoechoic to iso- or hyperechoic after 1 month of treatment is considered positive for patient improvement.

\section{Statistical Analysis}

Statistical analyses were performed using the SPSS for Windows, version 23.0 software. Descriptive statistics and frequency analysis were performed for categorical variables using counts and percentages, and the minimum, maximum, mean, and standard deviation are presented for the numerical variables. We performed the paired $t$ - test to compare the pretreatment and post-treatment findings within the groups; the McNemar test was used for categorical data. The crosstab chisquare test was used for categorical data to assess the differences among the 2 groups. A p value of $<0.05$ was considered statistically significant.

\section{Results}

A total of 54 patients were included in the study, 8 of them were missed during treatment sessions. 46 patients fulfilled the inclusion criteria 3 males (6.52\%) and 43 females (93.48\%) were included in the study, the age of patients ranged from 26 to 59 (mean 48.43 \pm 9.543 ) for ESWT and 22 to 62 (mean $46.09 \pm 10.126$ ) for laser group. Side effects were not observed in any of the patients. The demographic data and baseline clinical characteristics of the patients are listed in (Table 1).

Table 1: Demographic and baseline clinical characteristics of the patients

\begin{tabular}{|c|c|c|c|c|}
\hline \multicolumn{2}{|l|}{ Variables } & ESWT group $(n=23)$ & Laser group $(n=23)$ & $\mathrm{P}$ \\
\hline \multicolumn{2}{|l|}{ Age (Years) } & $46.0 \pm 10.2$ & $46.4 \pm 10$ & 0.913 \\
\hline Gender & $\begin{array}{l}\text { Male } \\
\text { Female }\end{array}$ & $\begin{array}{l}1(8.7 \%) \\
22(91 \%) \\
\end{array}$ & $\begin{array}{c}2(4.3 \%) \\
21(95.7 \%)\end{array}$ & 0.221 \\
\hline Symptoms Duration (mos) & & $6.4 \pm 5.3$ & $5.7 \pm 3.5$ & 0.622 \\
\hline Activity level & $\begin{array}{l}\text { Active } \\
\text { Sedentary }\end{array}$ & $\begin{array}{c}17(73.9 \%) \\
6(26.1 \%) \\
\end{array}$ & $\begin{array}{c}18(78.3 \%) \\
5(21.7 \%)\end{array}$ & 0.73 \\
\hline \multicolumn{2}{|l|}{ Body mass index } & $29.5 \pm 5.0$ & $32.8 \pm 5.3$ & 0.06 \\
\hline \multicolumn{2}{|l|}{ Calcaneal spur } & $18(78.3 \%)$ & $19(82.6 \%)$ & 0.71 \\
\hline \multicolumn{2}{|l|}{ Bilateral cases } & $4(17 \cdot 3 \%)$ & $3(13.0 \%)$ & 0.412 \\
\hline Tender point & $\begin{array}{l}\text { Central } \\
\text { Diffuse } \\
\text { Medial }\end{array}$ & $\begin{array}{c}3(13 \%) \\
5(21.7 \%) \\
15(65.2 \%)\end{array}$ & $\begin{array}{c}7(30.4 \%) \\
3(13 \%) \\
13(65.5 \%)\end{array}$ & 0.326 \\
\hline \multicolumn{2}{|l|}{ VAS pre intervention } & $8.2 \pm 1.5$ & $8.5 \pm 0.8$ & 0.366 \\
\hline \multicolumn{2}{|l|}{ FFI score pre intervention } & $118 \pm 14.9$ & $118.8 \pm 9.6$ & 0.788 \\
\hline \multicolumn{2}{|c|}{ Plantar fascia thickness pre intervention } & $0.5 \pm 0.09$ & $0.5 \pm 0.08$ & 0.664 \\
\hline
\end{tabular}

Data are presented as mean \pm standard deviation (SD) or no. (\%), Significant p-value $\leq 0.05$, Paired t-test, Chi-Square 
No significant differences were found in age, body mass index (BMI), or symptom duration among the 2 groups ( $p>0.05$ ) before treatment. Also, no significant differences were found in the initial parameters as determined using the VAS, FFI and PF thickness by US among the 2 groups ( $p$ $>0.05$ ). In both groups, significant differ- ences were found between the pre and post-treatment clinical values. The VAS score had significantly decreased and the FFI scores had significantly improved after treatment in the 2 groups ( $\mathrm{p}<0.001$, Tables 2 and 3). Mean FFI subscales pre and post intervention in both groups is present in table 4.

Table 2: Mean VAS score pre and post intervention

\begin{tabular}{|l|c|c|c|c|}
\hline Patients & $\begin{array}{c}\text { Pre-Intervention } \\
\text { Mean } \pm \text { SD }\end{array}$ & $\begin{array}{c}\text { Post Intervention } \\
\text { Mean } \pm \text { SD }\end{array}$ & $\begin{array}{c}\text { \% Improvement } \\
\text { in VAS }\end{array}$ & P-value \\
\hline ESWT $(n=23)$ & $8.2 \pm 1.5$ & $3.6 \pm 2.0$ & $40.0 \%$ & $\leq 0.01^{* *}$ \\
\hline Laser $(n=23)$ & $8.5 \pm 0.8$ & $2.8 \pm 1.3$ & $57.4 \%$ & $\leq 0.01^{* *}$ \\
\hline
\end{tabular}

*Significant $p$-value $\leq 0.05 * *$ highly significant $p$-value $\leq 0.001$

Table 3: Mean FFI pre and post intervention

\begin{tabular}{|l|c|c|c|c|}
\hline Patients & $\begin{array}{c}\text { Pre-Intervention } \\
\text { Mean } \pm \text { SD }\end{array}$ & $\begin{array}{c}\text { Post Intervention } \\
\text { Mean } \pm \text { SD }\end{array}$ & $\begin{array}{c}\text { \% Improvement } \\
\text { in FFI }\end{array}$ & P-value \\
\hline ESWT $(n=23)$ & $118.0 \pm 14.9$ & $60.5 \pm 18.8$ & $45.4 \%$ & $<0.001^{* *}$ \\
\hline Laser $(n=23)$ & $118.8 \pm 9.6$ & $57.6 \pm 8.3$ & $50.9 \%$ & $<0.001^{* *}$ \\
\hline
\end{tabular}

*Significant $p$-value $\leq 0.05 * *$ highly significant $p$-value $\leq 0.001$

Table 4: Mean FFI subscales pre and post intervention

\begin{tabular}{|l|c|c|c|c|c|c|}
\hline & \multicolumn{2}{|c|}{ Pre intervention (mean \pm SD) } & \multicolumn{2}{c|}{ Post intervention (mean \pm SD) } \\
\hline Patients & $\begin{array}{c}\text { Pain } \\
\text { subscale }\end{array}$ & $\begin{array}{c}\text { Disability } \\
\text { subscale }\end{array}$ & $\begin{array}{c}\text { Activity } \\
\text { Limitation } \\
\text { subscale }\end{array}$ & Pain subscale & $\begin{array}{c}\text { Disability } \\
\text { subscale }\end{array}$ & $\begin{array}{c}\text { Activity } \\
\text { Limitation } \\
\text { subscale }\end{array}$ \\
\hline ESWT $(\mathrm{N}=23)$ & $42.9 \pm 3.8$ & $68.3 \pm 10.0$ & $6.8 \pm 1.9$ & $21.1 \pm 5.3$ & $35.7 \pm 11.6$ & $3.7 \pm 4.6$ \\
\hline LLLT $(\mathrm{N}=23)$ & $42.8 \pm 3.6$ & $68.5 \pm 5.7$ & $7.1 \pm 1.1$ & $22.4 \pm 2.0$ & $33.8 \pm 5.8$ & $1.5 \pm 1.9$ \\
\hline
\end{tabular}

A significant decrease was detected in the thickness of the plantar fascia in both groups after treatment. The ESWT group showed a mean plantar fascia thickness of $0.53 \pm 0.09 \mathrm{~mm}$ at the baseline visit and a significant improvement after treatment $0.33 \pm 0.06 \mathrm{~mm} ;(p \leq 0.01$, Figure 1). The LLLT group showed a mean plantar fascia thickness of $0.54 \pm 0.08$ at the baseline visit and a significant improvement after treatment $0.35 \pm 0.06 \mathrm{~mm} ;(\mathrm{p} \leq 0.01$, Figure $2)$. The percentage of improvement in plantar fascia thickness was $37.1 \%$ in ESWT group and about $35.1 \%$ in LLLT group. A significant reduction was found in number of feet with hypo-echogenicity after treatment $(p<0.05)$ in both groups (Table 5). The improvement of PF regarding VAS, FFFI, plantar fascia thickness and echogenicity after one month of treatment in the ESWT and LLLT groups is presented in (table 6). LLLT proved significantly superior to ESWT therapy in pain relief $(p=0.02)$, but no significant difference between both groups in $\mathrm{FFI}$ improvement $(p=0.26)$. No significant difference between the two treatment modalities in both groups regarding the decrease in plantar fascia thickness and improvement in echogenicity by US was found (Table 7). 


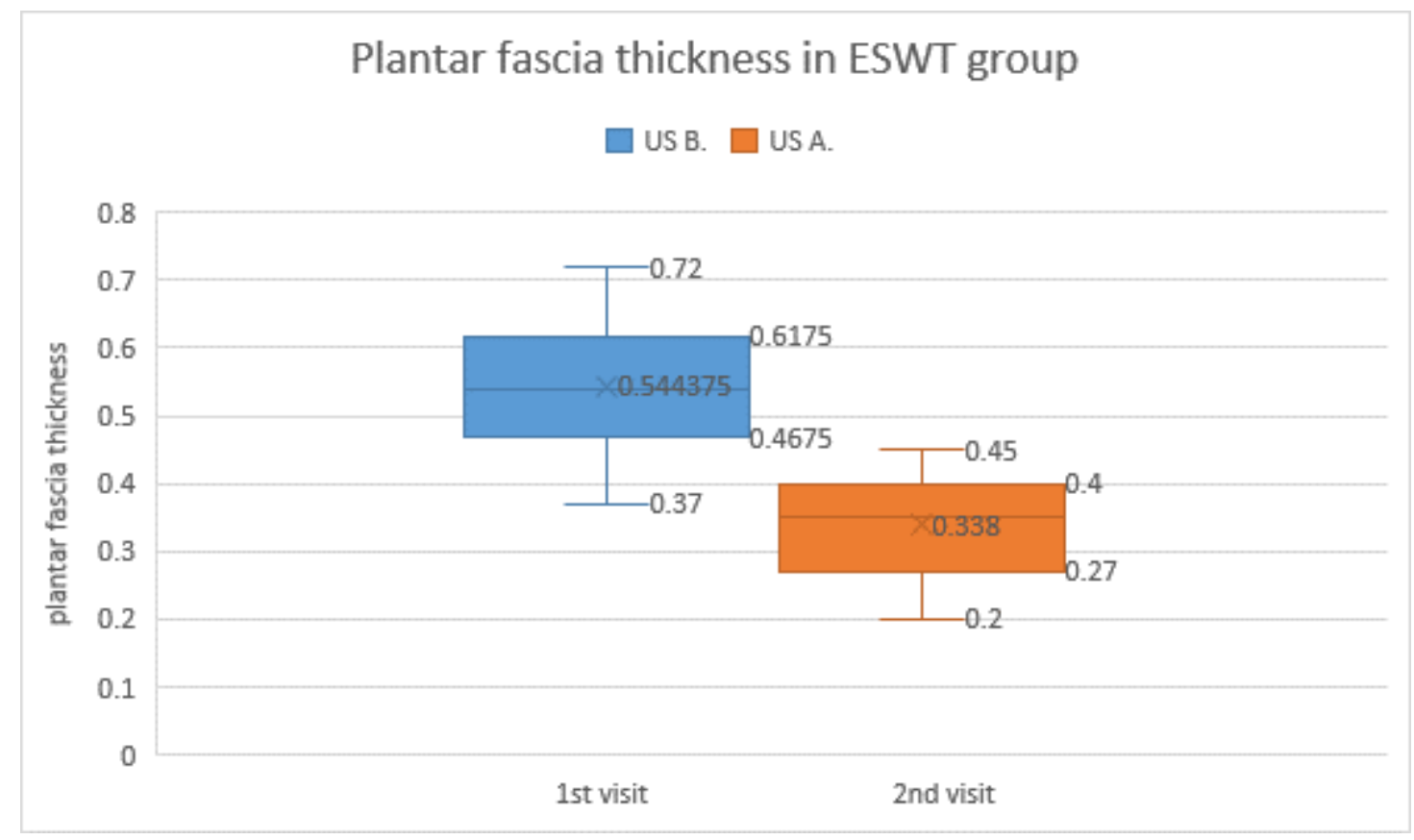

Figure 1: Plantar fascia thickness pre and post intervention in the ESWT group

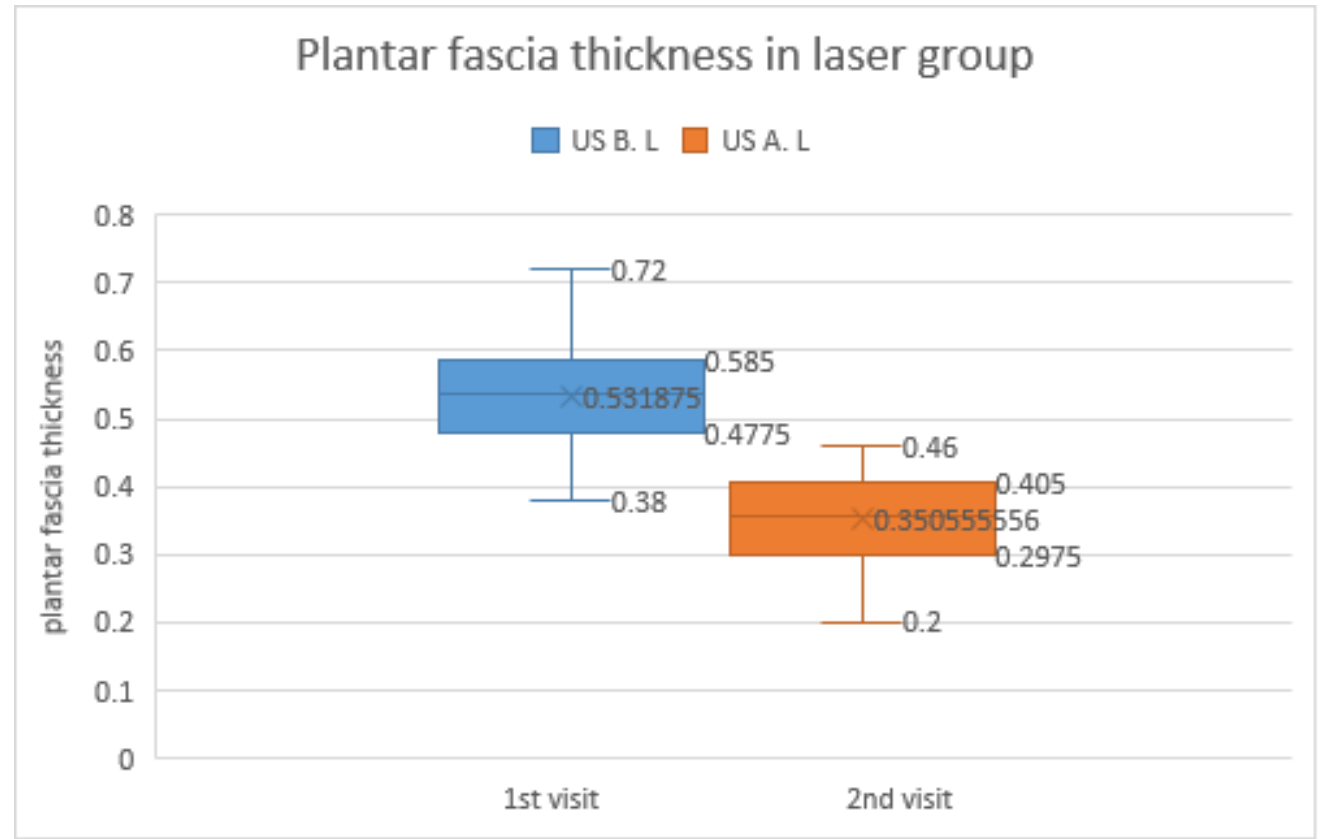

Figure 2: Plantar fascia thickness pre and post intervention in the LLLT group

\section{Discussion}

Plantar fasciitis is a common disorder that occurs in about $10 \%$ of the population. Commonly, patients present with pain on the plantar aspect of the foot at the attachment of the plantar fascia to the medial tubercle of the calcaneal bone ${ }^{(20)}$. Though different treatment modalities have been recommended, no strong evi- 
dence is available to support the use of any of the common strategies ${ }^{(21)}$. Most of the studies that investigate the effectiveness of PF treatments were frequently designed as placebo-controlled trials, and the number of studies comparing differ- ent treatment modalities has been rather limited(16). So, the aim of this study was to compare the efficacy of ESWT versus LLLT in the management of PF using diagnostic US as an objective method for assessment.

Table 5: plantar fascia echogenicity in both groups pre and post intervention

\begin{tabular}{|l|c|c|c|c|}
\hline \multicolumn{2}{|c|}{ LLLT group } & \multicolumn{2}{c|}{ ESWT group } \\
\hline Iso or hyperechoic & $12(52.2 \%)$ & $22(95.7 \%)$ & $8(37.6 \%)$ & $19(82.6 \%)$ \\
\hline Hypoechoic & $11(47.8 \%)$ & $1(4.3 \%)$ & $15(62.4 \%)$ & $4(17.4 \%)$ \\
\hline P value & \multicolumn{2}{|c|}{$.018^{*}$} & \multicolumn{2}{c|}{$0.006^{* *}$} \\
\hline
\end{tabular}

*Significant $p$-value $\leq 0.05$, **highly significant $p$-value $\leq 0.01$, McNemar test

Table 6: Improvement in VAS, FFI, plantar fascia thickness, and echogenicity in ESWT and LLLT

\begin{tabular}{|l|c|c|}
\hline & ESWT $(\mathrm{N}=23)$ & LLLT $(\mathrm{N}=23)$ \\
\hline VAS improvement $\geq 30 \%$ & $21(90.5 \%)$ & $23(100 \%)$ \\
\hline FFI improvement $\geq 50 \%$ & $13(56.5 \%)$ & $18(69.2 \%)$ \\
\hline FFI improvement $<50 \%$ & $10(43.4 \%)$ & $5(30.7 \%)$ \\
\hline Plantar Fascia thickness $\leq .4 \mathrm{~cm}$ & $20(86.9 \%)$ & $18(73.0 \%)$ \\
\hline Plantar Fascia thickness increased & 0 & $1(3.8 \%)$ \\
\hline Thickness improvement $\geq .4 \mathrm{~cm}$ & $3(13.0 \%)$ & $4(23.0 \%)$ \\
\hline $\begin{array}{l}\text { Improved plantar fascia } \\
\text { (from hypo to iso or hyper-echoic) }\end{array}$ & $11(47.8 \%)$ & $10(43.4 \%)$ \\
\hline
\end{tabular}

Table 7: Comparison between ESWT and LLLT groups regarding degree of improvement in VAS, FFI, plantar fascia thickness and echogenicity after treatment

\begin{tabular}{|l|c|c|c|}
\hline Mean \pm SD & ESWT & Laser & P value \\
\hline Decrease in VAS & $4.6 \pm 2.0$ & $5.6 \pm 1.3$ & 0.029 \\
\hline Decrease in FFI & $57.2 \pm 14.0$ & $61.1 \pm 6.7$ & 0.264 \\
\hline Decrease in Plantar fascia Ithickness in mm & $0.2 \pm 0.08$ & $0.1 \pm 0.09$ & 0.885 \\
\hline $\begin{array}{l}\text { echogenicity Change (from } \\
\text { hypoechoic to iso or hyperechoic) }\end{array}$ & $\begin{array}{c}\text { No. } \% \\
11(47.8 \%)\end{array}$ & $\begin{array}{c}\text { No. } \% \\
10(43.4 \%)\end{array}$ & 0.34 \\
\hline
\end{tabular}

*Significant $p$-value $\leq 0.05$, **highly significant $p$-value $\leq 0.01$, McNemar test

Up to our knowledge this is the first study to use diagnostic US to compare the efficacy of ESWT and LLLT in the management of PF. The findings from the present study showed that both ESWT and LLLT are effective methods for management of PF regarding pain relief, improvement of foot function, and reduction of plantar fascia thickness by ultrasonography. However, LLLT proved significantly superior to ESWT therapy in pain relief $(p=$ 0.029), but no significant difference be- tween both groups in FFI, plantar fascia thickness or change in echogenicity ( $p>0.05$ ). Similar findings were reported by a recent study done by Cinar et al in $2018^{(22)}$ that compared the effectiveness of ESWT and LLLT to relieve pain in patients with chronic PF. Patients were randomized into three groups: LLLT $(n=24)$, ESWT $(n=25)$, and control $(n=17)$. All participants received a home exercise program with orthotic supports. Cinar et al found that a substantial percentage of 
patients in the group of LLLT (79\%) showed significant improvement in pain compared to the ESWT group (61\%) and the control group (50\%) at three months after treatment ${ }^{(22)}$. However, Cinar et al didn't measure plantar fascia thickness which is an objective method to assess the efficacy of treatment. They only used Numerical Rating Scale for pain and FFIpain subscale to assess the effectiveness of both modalities ${ }^{(22)}$. Ulusoy et al in 2017 (16) used magnetic resonance imaging (MRI) to compare the effectiveness of ESWT, LLLT, and US on the relief of pain, foot function, and plantar fascia thickness, Sixty patients with chronic PT were randomized into 3 groups $(n=20)$ in each group. They reported that plantar fascia thickness had decreased significantly on MRI in all the 3 groups. The success rate of treatment was $70.6 \%$ in the LLLT group, $65 \%$ in the ESWT group, and $23.5 \%$ in the US group at 1 month after treatment. LLLT and ESWT caused higher improvement in foot function and pain. Comparable to our results, Ulusoy et al showed that LLLT was significantly superior to ESWT in reducing pain ${ }^{(16)}$. Although MRI is a valid method to assess plantar fascia thickness, it is a costly tool to be used for follow up. Whereas US is considered a reliable and inexpensive imaging technique for assessing $\mathrm{PF}$ thickness ${ }^{(23)}$. The efficacy of ESWT in the treatment of PF was investigated in a large number of randomized controlled trials $(R C T)^{(16,24,25)}$ In the present study, the ESWT group showed significant improvement in all clinical parameters after one month of treatment, $56.52 \%$ of the patients reported $a \geq 50 \%$ improvement in the $\mathrm{FFI}, 90.5 \%$ of the patients reported $\mathrm{a} \geq$ $30 \%$ reduction in the pain score and the plantar fascia thickness was reduced in $86.95 \%$ of the patients. A meta-analysis was done in 2013 to investigate the efficacy of ESWT compared to placebo in the treatment of chronic PF and reported a >
$60 \%$ reduction in pain scores and improvement in Roles-Maudsley score (RMS) which is a patient-administered scoring system regarding activity limitations. The meta-analysis showed that ESWT was significantly superior to placebo and they recommended ESWT for patients not improving after three months of other non-operative treatment modalities ${ }^{(26)}$. Conversely, some RCT did not show the superiority of ESWT to placebo. In 2003 Haake et al ${ }^{(27)}$ randomized 272 patients into three sessions of ESWT or sham ESWT. The success of treatment was defined as achieving an RMS of 1 or 2. The success rate was nearly similar in the two groups at 12 weeks ( $34 \%$ for ESWT versus $30 \%$ for placebo) and at one year of follow-up ( $81 \%$ for ESWT versus $76 \%$ for placebo) ${ }^{(27)}$. Although ESWT has gained higher popularity among researchers and clinicians for its effectiveness in improving PF symptoms we didn't find additive effect of ESWT over LLLT. LLLT therapy is considered a safe modality and well tolerated by the patients. Moreover, It is easier to use, cheaper than ESWT, and causes less pain for the patient ${ }^{28)}$. The percentage of pain reduction using LLLT has been reported to range from $20 \%$ to $80 \%$ by different studies ${ }^{(29,30)}$ The variability in the results of the studies may be due to the differences in the type of laser, frequency and the dose used for treatment ${ }^{(22)}$. In this study we used $830 \mathrm{~nm}$ of laser light, at a frequency of $10.0 \mathrm{~Hz}$ leading to a total dose of $27.0 \mathrm{~J} / \mathrm{cm} 2$. the percentage of pain reduction was $57.4 \%$ and nearly all the patients in the LLLT group exhibited $\geq 30 \%$ improvement in the VAS score. About $69 \%$ of the patients experienced $\geq 50 \% \mathrm{im}$ provement in the FFI and the plantar fascia thickness decreased significantly after one month of treatment similar to the findings from Macias et al(30). These results have proved that LLLT is a reliable and efficient treatment modality for 
chronic PF. In literature, it is generally accepted that plantar fascia thickness of more than $4 \mathrm{~mm}$ would be abnormal and it is considered as a well-established sonographic criterion for the diagnosis of plantar fasciitis(31,32). In the present study both ESWT and LLLT resulted in a significant decrease in plantar fascia thickness $(p \leq 0.01)$. This is similar to the results of the study done by Saber(5) where ESWT group had also showed significant decrease in plantar fascia thickness from 5.9 \pm 0.5 before treatment to a mean of $3.3 \pm 0.4$ after treatment $(p<0.001)$ and with the results of Kiritsi et al(33) who demonstrated a reduction in plantar fascia thickness in LLLT and placebo groups. Furthermore, when the difference in plantar fascia thickness was compared between the LLLT group and the placebo group, the change was statistically significant $(p=0.007)^{(33)}$. In the present study although the improvement in the thickness of the plantar fascia was greater in the ESWT than the LLLT group, this was not statistically significant $(p=0.885)$. Fabrikant and Park(34) measured the plantar fascia thickness by ultrasound before and after treatment with injection and biomechanical correction and found no correlation between the reduction in plantar fascia thickness and the reduction in patients reported level of pain. In the present study, we have confirmed these findings by verifying no correlation between the reduction in the plantar fascia thickness oand improvement in VAS score $(p=0.6)$ or the improvement in FFI $(p=0.3)$. However, Ulusoy et al measured the plantar fascia thickness before and after treatment using MRI and found correlation between the plantar fascia thickness reduction and pain reduction. Also, plantar fascia thickness reduction was greater for patients with a good foot function after treatment ${ }^{(16)}$ The inconsistency in the results between these studies may be due to the difference in the method used to measure the thickness of the plantar fascia. The normal plantar fascia is hyperechoic and evenly fibrillary. Conversely, a hypoechoic plantar fascia is a frequent ultrasonographic finding in $\mathrm{PF}^{(32)}$. Our results regarding the qualitative assessment of $\mathrm{PF}$ by echogenicity revealed that the change from hypoechoic to hyper or isoechoic plantar fascia was statistically significant 11 (47.8\%) in ESWT and 10 (43.4\%) in laser group ( $p=0.006$ and 0.018 ) respectively, but there was no significant difference between both groups $(p=0.34)$. Up to our knowledge, no studies have examined the change in echogenicity in PF after the treatment with ESWT or LLLT yet. However, Moustafa et al ${ }^{(18)}$ showed statistically significant reduction in number of hypoechoic feet after dexamethasone injection in patients with PF $(p<0.05)^{(18)}$. Even though the present study supports the short-term effectiveness of ESWT and LLLT in the management of PF with a better improvement in pain in LLLT group, these findings should be interpreted in the light of some limitations. First, the follow-up period of PF might have been better extended to 6 months or more to understand the long- term effects of both modalities. Second, a control group with only exercises might have added more validity to the results by comparing the results to the natural progression of the disease.

\section{Conclusion}

Both ESWT and LLLT are effective methods for management of PF regarding improvement in pain scores, foot function and plantar fascia thickness. However, LLLT was more superior to ESWT regarding pain relief.

\section{References}

1. Jastifer JR, Catena F, Doty JF, Stevens 
F and Coughlin MJ 2014 Low-Level Laser Therapy for the Treatment of Chronic Plantar Fasciitis: A Prospective Study. Foot Ankle Int. 35 566-71

2. Riddle DL, and Schappert SM. 2004 Volume of ambulatory care visits and patterns of care for patients diagnosed with plantar fasciitis: a national study of medical doctors. Foot ankle Int. 25 303-10

3. Goff JD and Crawford R 2011 Diagnosis and treatment of plantar fasciitis. Am. Fam. Physician 84 676-82

4. Thomas JL, Christensen JC, Kravitz SR, et al. 2010 The Diagnosis and Treatment of Heel Pain: A Clinical Practice Guideline-Revision $2010 \mathrm{~J}$. Foot Ankle Surg. 49

5. Saber N 2012 Ultrasound guided local steroid injection versus extracorporeal shockwave therapy in the treatment of plantar fasciitis Alexandria J. Med. 48 35-42

6. Delisa J 2010 DeLisa's Physical Medicine \& Rehabilitation

7. Yucel I 2010 Comparison of High-Dose Extracorporeal Shockwave Therapy and Intralesional Corticosteroid Injection in the Treatment of Plantar Fasciitis 100 105-10

8. Hammer DS, Adam F, Kreutz A, Rupp S, Kohn D and Seil R 2005 Ultrasonographic evaluation at 6-month followup of plantar fasciitis after extracorporeal shock wave therapy. Arch. Orthop. Trauma Surg. 125 6-9

9. Samarbakhsh A, Shojaeei M and Hami M 2013 Efficacy of low level laser therapy (LLLT) in lumbar discopathic pain (randomized controlled study) Pain Res. Manag. 18 e 41

10. Cotler HB, Chow RT, Hamblin MR and Carroll J. 2015. The Use of Low Level Laser Therapy (LLLT) For Musculoskeletal Pain. MOJ Orthop Rheumatol. 2(5). pii: 00068. Epub.

11. Lubart $\mathrm{R}$, Wollman $\mathrm{Y}$, Friedmann $\mathrm{H}$, Rochkind S. and Laulicht I. 1992 Effects of visible and near-infrared lasers on cell cultures J. Photochem. Photobiol. B Biol. 12 305-10
12. Gollwitzer H, Saxena A, DiDomenico LA, et al. 2015 Clinically relevant effectiveness of focused extracorporeal shock wave therapy in the treatment of chronic plantar fasciitis: a randomized, controlled multicenter study J Bone Jt. Surg Am 97 701-8

13. Malay DS, Pressman MM, Assili A, Kline J T, York S, Buren B, Heyman E R, Borowsky P and Lemay $C$ et al. 2006 Extracorporeal Shockwave Therapy Versus Placebo for the Treatment of Chronic Proximal Plantar Fasciitis: Results of a Blinded, Multicenter Intervention Trial

14. Zhiyun L, Tao J, and Zengwu S. 2013 Meta-analysis of high-energy extracorporeal shock wave therapy in recalcitrant plantar fasciitis Swiss Med Wkly;143: w13825.

15. Thomson CE, Crawford F, and Murray GD. 2005: The effectiveness of extra corporeal shock wave therapy for plantar heel pain: a systematic review and meta-analysis. BMC Musculoskelet. Disord. 619

16. Ulusoy A, Cerrahoglu L and Orguc $S$ 2017 Magnetic Resonance Imaging and Clinical Outcomes of Laser Therapy, Ultrasound Therapy, and Extracorporeal Shock Wave Therapy for Treatment of Plantar Fasciitis: A Randomized Controlled Trial J. Foot Ankle Surg. 56 762-7

17. Budiman-Mak E Conrad K Roach K. 1991 The foot function index: A measure of foot pain and disability $\mathrm{J}$. Clin. Epidemiol. 44 561-70

18. Moustafa A, Hassanein E and Foti $C$ 2015 Objective assessment of corticosteroid effect in plantar fasciitis: additional utility of ultrasound. Muscles. Ligaments Tendons J. 5 28996

19. Sabir N, Demirlenk S, Yagci B, Karabulut N, and Cubukcu S. 2005 Clinical utility of sonography in diagnosing plantar fasciitis J. Ultrasound Med. 24 1041-8

20. Ahmad J, Karim A, Daniel JN. 2016 Relationship and Classification of 
Plantar Heel Spurs in Patients With Plantar Fasciitis. Foot Ankle Int. ; 37(9):994-1000

21. Crawford F and Thomson C E 2003 Interventions for treating plantar heel pain Cochrane Database of Systematic Reviews ed F Crawford (Chichester, UK: John Wiley \& Sons, Ltd)

22. Cinar E, Saxena S and Uygur F. 2018 Combination Therapy Versus Exercise and Orthotic Support in the Management of Pain in Plantar Fasciitis: A Randomized Controlled Trial Foot Ankle Int. 39 406-14

23. Mohseni-Bandpei MA, Nakhaee $M$, Mousavi ME, Shakourirad A, Safari MR and Vahab Kashani R. 2014 Application of ultrasound in the assessment of plantar fascia in patients with plantar fasciitis: A systematic review Ultrasound Med. Biol. 40 (8): 1737-54.

24. Sun J, Gao F, Wang Y, Sun W, Jiang B and Li Z 2017 Extracorporeal shock wave therapy is effective in treating chronic plantar fasciitis: A metaanalysis of RCTs. Medicine (Baltimore). 96 e6621

25. Eslamian F, Shakouri SK, Jahanjoo F, Hajialiloo M and Notghi F. 2016. Extra Corporeal Shock Wave Therapy Versus Local Corticosteroid Injection in the Treatment of Chronic Plantar Fasciitis, a Single Blinded Randomized Clinical Trial. Pain Med. 17(9):1722-31.

26. Aqil A, Siddiqui M, Solan M. et al. 2013 Extracorporeal Shock Wave Therapy Is Effective In Treating Chronic Plantar Fasciitis: A Meta-analysis of RCTs. Clin. Orthop. Relat. Res. 471 3645-52

27. Haake $M$, Buch $M$, Schoellner $C$ et al. 2003 Extracorporeal shock wave therapy for plantar fasciitis: randomised controlled multicentre trial. BMJ. 327 (7406):75.

28. Hashmi JT, Huang YY, Osmani BZ, Sharma SK, Naeser MA, and Hamblin MR. 2010 Role of low-level laser therapy in neurorehabilitation PM R. 2(12 Suppl 2):S292-305.

29. Jastifer JR, Catena F, Doty JF, Stevens F, and Coughlin MJ. 2014 Low-Level
Laser Therapy for the Treatment of Chronic Plantar Fasciitis: A Prospective Study Foot Ankle Int. 35 566-71

30. Macias D, Coughlin M, Zang K, Stevens F, Jastifer J. et al. 2015 Low-Level Laser Therapy at $635 \mathrm{~nm}$ for Treatment of Chronic Plantar Fasciitis: A Placebo-Controlled, Randomized Study J. Foot Ankle Surg. 54 768-72

31. Karabay N, Toros T and Hurel C 2007 Ultrasonographic Evaluation in Plantar Fasciitis J. Foot Ankle Surg. 46 442-6

32. Gibbon WW and Long G 1999 Ultrasound of the plantar aponeurosis (fascia). Skeletal Radiol. 28 21-6

33. Kiritsi O, Tsitas K, Malliaropoulos $\mathrm{N}$ and Mikroulis G. 2010; Ultrasonographic evaluation of plantar fasciitis after low-level laser therapy: results of a double-blind, randomized, placebocontrolled trial Lasers Med. Sci. 25 275-81

34. Fabrikant JM and Park TS. 2011 Plantar fasciitis (fasciosis) treatment outcome study: Plantar fascia thickness measured by ultrasound and correlated with patient self-reported improvement Foot 21 79-83 
